\section{APPLIED PHYSICS}

\section{Martian dust blower}

New J. Phys. 19, 063006 (2017)

Your Earthly carpet sweeper won't do the job in the low-pressure, $\mathrm{CO}_{2}$-dominant atmosphere on Mars. But Catalin Ticoş and colleagues have now shown how to build a Mars-proof dirt broom, which can be used for removing sand and dust from equipment stationed on the Martian surface.

The authors' experimental setup involved a coaxial plasma gun, capable of producing dense pulsed plasma jets, directed perpendicularly to the area to be cleaned. As a test surface, they used an array of photovoltaic cells, covered with a powder retrieved from volcanic ash, which mimicked Martian surface soil.

Ticoş et al. measured the efficiency of their cleaning method in terms of how the voltage delivered by the cells increased during operation. An analysis of the plasma jets in a $\mathrm{CO}_{2}$ environment at the same pressure as that on Mars's surface revealed an average plasma plume speed several orders larger than the planet's typical wind speeds - implying that the plasma broom would indeed succeed in the Martian environment.

\section{STATISTICAL PHYSICS}

\section{Dialectic magnetism}

Phys. Rev. X (in the press); preprint at http://arxiv.org/abs/1703.00533

We're all familiar with the idea that two people from the same country, living a short distance apart, might speak very different dialects. Intuitively, you'd expect that history and geography have each played a role in shaping the boundary between their dialects, but is it possible to formalize this hunch?

To address this question, James Burridge resorted to statistical mechanics, deriving macroscopic equations for language evolution. In his theory, speakers adopt the language variant that they hear the most: this local alignment, like spins in a ferromagnet, leads to the emergence of domains bound by domain walls - or 'isoglosses' in the linguist's lingo. Taking into account the varying population density, the boundaries between domains spontaneously bend around relevant geographical features, reflecting the stronger influence of cities and the linguistic barrier posed by mountains or other scarcely populated areas.

The agreement between the model's predictions and real data is a reminder of the efficacy of coarse-grained descriptions based on simple principles - in this case, surface tension minimization - to capture the salient features of complex systems.

\section{ANYONS}

\section{Not just another statistic}

Phys. Rev. Lett. 118, 227201 (2017)

Particles are usually categorized as being either bosons or fermions, obeying Bose-Einstein or Fermi-Dirac statistics. When dealing with two-dimensional systems, however, quasiparticles known as anyons can emerge, whose statistics lie somewhere between the two. As they can exhibit nonAbelian behaviour, researchers are looking to exploit anyons for topological quantum computation. But experimentally, just seeing these quasiparticles is far from easy. Siddhardh Morampudi and colleagues have now shown that signatures of anyons could be detected using established techniques such as neutron scattering or tunnelling spectroscopy.

In certain materials, scattering processes can create fractionalized quasiparticle excitations. Morampudi et al. showed that if such quasiparticles have anyonic statistics, and you're near the threshold energy for their excitation, then the scattering crosssections should exhibit a distinct power

\section{BIOMIMETICS}

\section{A sticky job for suckers}

Nature 546, 396-400 (2017)

How do you build an adhesive material that performs well underwater? Start by asking an octopus. Everyone's favourite cephalopod excels at using the adhesive power of the suckers on its tentacles to get around. Sangyul Baik and colleagues took inspiration from this design to fabricate a reversible adhesion system that works well in both wet and dry conditions. Their simulacrum even managed to heft a weight (here, a silicon wafer) through water and air much like an octopus transporting its prey.

Baik et al. developed a technique involving a patterned silicon structure that they used as a master for their adhesive polymer-based material, which they then tested by sticking it to silicon wafers, glass and skin under varying degrees of moisture. They explained the material's remarkable underwater performance with a model combining suction and capillary stresses - and highlighting the role of geometry in optimizing cohesive forces. Success in the case of adhesion to pig skin indicates that the material may be applied to wound healing. $\quad A K$ law whose exponent depends only on the statistics of the anyons. This threshold spectroscopy-based approach provides a clear route for looking for anyonic excitations in topologically ordered materials such as gapped quantum spin liquids. But these ideas may also be applicable to ultracold atoms in optical lattices - greatly expanding the list of candidate systems to explore.

\section{METEORITICS}

\section{Silica puzzle}

Nat. Commun. 8, 15647 (2017)

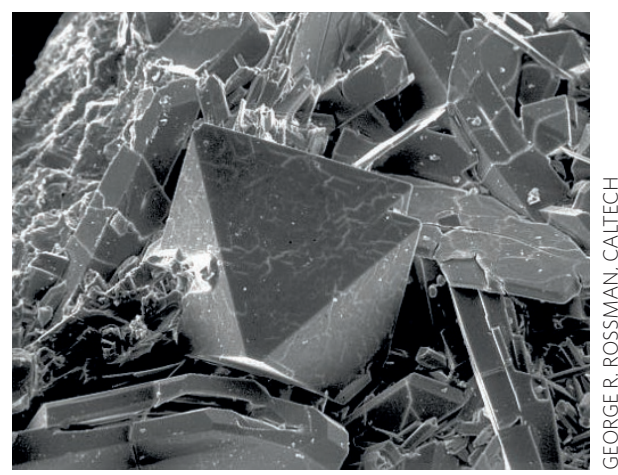

On Earth, the most common form of silicon dioxide is quartz. But in planetary dust, meteorites and lunar rocks, the mineral predominantly has a different structure, known as cristobalite (pictured). In meteorites, cristobalite comes mixed with yet another form of silica mineral called seifertite. But seifertite forms at very high pressures (over $80 \mathrm{GPa}$ ), implying a massive meteorite impact, whereas cristobalite requires much lower pressures. So the peculiar coexistence of the two structures in the same meteorite has intrigued researchers. And now, Ana Černok and colleagues have used highpressure conditions and X-ray diffraction to understand how the two types of silica are formed.

Černok and co-workers found that when cristobalite is compressed uniformly around $11 \mathrm{GPa}$, it turns into a high-pressure phase called cristobalite X-I, which reverts back upon decompression. But when the pressure is uneven, cristobalite transforms into seifertite at a much lower pressure $(50 \mathrm{GPa})$ than it would from ordinary silica. In meteorites, impact shock waves create areas with even and uneven compression where cristobalite and seifertite, respectively, can form. This also suggests that meteorites containing sefertite need not have necessarily suffered a dramatic impact.

Written by Luke Fleet, Iulia Georgescu,

Abigail Klopper, Federico Levi and Bart Verberck. 